\title{
Effect of a national primary care pay for performance scheme on emergency hospital admissions for ambulatory care sensitive conditions: controlled longitudinal study
}

\author{
(c) (1) (9) OPEN ACCESS
}

\begin{abstract}
Mark J Harrison honorary senior research fellow, and assistant professor ${ }^{123}$, Mark Dusheiko senior research fellow, and assistant professor ${ }^{45}$, Matt Sutton professor ${ }^{1}$, Hugh Gravelle professor ${ }^{2}$, Tim Doran professor ${ }^{7}$, Martin Roland professor ${ }^{6}$
\end{abstract}

\begin{abstract}
${ }^{1}$ Manchester Centre for Health Economics, Institute of Population Health, University of Manchester, UK; ${ }^{2}$ Faculty of Pharmaceutical Sciences, University of British Columbia, Vancouver, BC, Canada; ${ }^{3}$ Centre for Health Evaluation and Outcome Sciences, St Paul's Hospital, Vancouver, BC, Canada; ${ }^{4}$ Centre for Health Economics, University of York, York, UK; ${ }^{5}$ Institute for Health Economics and Management, University of Lausanne, Lausanne, Switzerland; ${ }^{6}$ Cambridge Centre for Health Services Research, University of Cambridge, Forvie Site, Robinson Way, Cambridge, CB2 0SR, UK; ${ }^{7}$ Department of Health Sciences, University of York, York, UK
\end{abstract}

\begin{abstract}
Objective To estimate the impact of a national primary care pay for performance scheme, the Quality and Outcomes Framework in England, on emergency hospital admissions for ambulatory care sensitive conditions (ACSCs).

Design Controlled longitudinal study.

Setting English National Health Service between 1998/99 and 2010/11. Participants Populations registered with each of 6975 family practices in England.

Main outcome measures Year specific differences between trend adjusted emergency hospital admission rates for incentivised ACSCs before and after the introduction of the Quality and Outcomes Framework scheme and two comparators: non-incentivised ACSCs and non-ACSCs.

Results Incentivised ACSC admissions showed a relative reduction of $2.7 \%$ (95\% confidence interval $1.6 \%$ to $3.8 \%$ ) in the first year of the Quality and Outcomes Framework compared with ACSCs that were not incentivised. This increased to a relative reduction of $8.0 \%(6.9 \%$ to $9.1 \%$ ) in 2010/11. Compared with conditions that are not regarded as being influenced by the quality of ambulatory care (non-ACSCs), incentivised ACSCs also showed a relative reduction in rates of emergency admissions of $2.8 \%(2.0 \%$ to $3.6 \%)$ in the first year increasing to $10.9 \%(10.1 \%$ to $11.7 \%)$ by $2010 / 11$.

Conclusions The introduction of a major national pay for performance scheme for primary care in England was associated with a decrease in
\end{abstract}

emergency admissions for incentivised conditions compared with conditions that were not incentivised. Contemporaneous health service changes seem unlikely to have caused the sharp change in the trajectory of incentivised ACSC admissions immediately after the introduction of the Quality and Outcomes Framework. The decrease seems larger than would be expected from the changes in the process measures that were incentivised, suggesting that the pay for performance scheme may have had impacts on quality of care beyond the directly incentivised activities.

\section{Introduction}

Many countries have introduced pay for performance schemes to improve care for chronic conditions. Rather than directly rewarding improved patient outcomes, these schemes generally provide incentives to doctors for improving processes of care (for example, blood pressure checks for patients with hypertension) and intermediate outcomes (for example, cholesterol control in people with diabetes). Evaluations of pay for performance schemes have found limited impacts on processes of care,$^{1-3}$ including improvements in non-incentivised aspects of care for targeted patients. ${ }^{4}$ However, pay for performance schemes have not consistently reduced costs ${ }^{56}$ and are sometimes associated with unintended consequences and detrimental effects on quality of care for non-targeted patients. ${ }^{78}$ Even when improvements in processes of care are shown, they are often modest ${ }^{9}{ }^{10}$ and lacking in apparent impacts on patient outcomes. ${ }^{11}$ Although several studies have found associations 
between the introduction of pay for performance schemes and reduced hospital admissions, ${ }^{12-14}$ complications, ${ }^{15}{ }^{16}$ and mortality, ${ }^{17}$ a recent systematic review concluded that the effect of pay for performance schemes on patient outcomes was largely uncertain. ${ }^{18}$ Part of the reason for this uncertainty may be that the impact of pay for performance schemes depends on their context and design, ${ }^{19-22}$ and this has led to experimentation with new and more innovative types of contract. ${ }^{23} 24$

The implementation of a major pay for performance scheme- the Quality and Outcomes Framework-in all family practices in England provides a natural experiment to evaluate the impact of pay for performance on hospital admissions for ambulatory care sensitive conditions (ACSCs). ACSCs are conditions for which hospital admissions could be prevented or reduced through management of the acute episode in the community or by preventive care. In principle they are admissions that could be avoided by providing high quality primary care. Many conditions included in pay for performance schemes are ambulatory care sensitive so, if the schemes are successful in improving quality they should lead to a reduction in hospital admissions. The focus of UK policy makers has been to reduce emergency (unscheduled or unplanned) admissions for ACSCs because these represent a large and increasing proportion of healthcare costs. ${ }^{25}$

We studied emergency hospital admissions for ACSCs included in the Quality and Outcomes Framework ${ }^{26}$ using data for five years before and seven years after the introduction of the scheme. We compared rates of hospital admissions for these conditions with comparators that should not have been directly affected by the Quality and Outcomes Framework

scheme-namely, emergency admissions for ACSCs that were not directly incentivised by the Quality and Outcomes

Framework and emergency admissions that were not for ACSCs.

\section{Methods}

\section{Pay for performance scheme}

The Quality and Outcomes Framework was introduced in April 2004 and implemented simultaneously across all practices in the four countries of the United Kingdom, including England. We use data for England. The scheme links up to $25 \%$ of family practitioner income to performance on over 100 clinical and organisational quality indicators. For the clinical indicators, practices are awarded points based on the proportion of patients for whom targets are achieved between a lower achievement threshold (initially set at $25 \%$ for all indicators) and an upper threshold that varies from $50 \%$ to $90 \%$ according to the indicator. The maximum number of points available also varies by indicator, with more points allocated to intermediate outcomes indicators (for example, blood pressure control) than to monitoring indicators (for example, blood pressure measurement). Points are converted into payments to practices after adjustment for list size and disease prevalence. Ten chronic conditions were included in the original scheme, with financial incentives ranging from $£ 75(\$ 121 ; € 95)$ to $£ 4200$ per indicator for the average practice in the first year, and rewards increased by $68 \%$ in the second year. The scheme is periodically reviewed and quality indicators may be modified or removed, and novel indicators may be introduced covering existing or new conditions. ${ }^{27}$ By 2010/11 (the final year analysed in the study), 16 chronic conditions were included in the scheme.

\section{Sources of data}

Our primary data source was hospital episode statistics, a national database of admissions to hospitals in England, with information on the reason for admission and the family practices with which admitted patients are registered. We analysed data on all emergency admissions to National Health Service hospitals in England for the financial years (1 April to 31 March) $1998 / 99$ to $2010 / 11$, excluding admissions that were transfers between hospitals. Hospital episodes are recorded for each period patients are in the care of hospital consultants. We defined emergency admissions as being the first episode in a spell of care, coded as an emergency, and admitted from a source other than another hospital ward or outpatient clinic. Using ICD-10 (international classification of diseases, 10th revision) codes for the primary diagnosis, we distinguished between emergency admissions for ACSCs incentivised in the Quality and Outcomes Framework, ACSCs that were not incentivised in the Quality and Outcomes Framework, and non-ACSCs. We compared the changes in admissions for incentivised ACSCs before and after the introduction of the Quality and Outcomes Framework with changes in admissions for the other two groups of conditions, non-incentivised ACSCs and non-ACSCs.

\section{Inclusion and exclusion criteria}

We based our list of ambulatory sensitive conditions on those used for measuring system performance in the NHS. ${ }^{28} \mathrm{We}$ modified this list by excluding dental conditions (as these are not the responsibility of medical practitioners in the United Kingdom), influenza, and pneumonia (as influenza immunisation was incentivised to varying degrees during the study period), malnutrition related diabetes (which is specifically excluded from the pay for performance scheme), and febrile convulsions and eclampsia (as the focus of the quality indicators for epilepsy was on the management of long term epilepsy). We included a small number of diagnoses adapted from a previous study ${ }^{28}$ : ICD codes I25 (chronic ischaemic heart disease), I130 (hypertensive heart and renal disease with congestive heart failure), J04 (acute laryngitis and tracheitis), J44 (other chronic obstructive pulmonary disease), and N30.0, N30.8, and N30.9 (cystitis). We also included diabetic complications associated with hypoglycaemia. ${ }^{29}$ The supplementary file lists the conditions included in the analysis.

For incentivised ACSCs we included admissions with a primary diagnosis of the following conditions, which have been continually incentivised under the Quality and Outcomes Framework since the introduction of the scheme: asthma, coronary heart disease, congestive heart disease, chronic obstructive pulmonary disease, diabetes (separated into admissions for hyperglycaemia and hypoglycaemia), epilepsy, hypertension, and stroke. For non-incentivised ACSCs we included all remaining diagnostic categories of ACSCs, providing the relevant conditions were not targeted under the pay for performance scheme at any time during the study period. The five non-incentivised ACSCs with the highest admission rates in 2010/11 were ear, nose, and throat conditions; dehydration; cellulitis; pyelenophritis and urinary tract infection; and anaemia. Non-ACSCs consisted of all remaining admissions.

\section{Statistical analysis}

We aggregated admissions to the level of the family practice and excluded from the analysis those practices with a registered population smaller than 1000 (an average of 117 practices in each year). We used a balanced panel of practices appearing in all of the financial years-6975 different practices (mean population 6264 patients). Admission rates were calculated as the total number of admissions divided by the total registered practice population for that year. 
We analysed data for five years before (1998/99 to 2002/03) and seven years after (2004/05 to 2010/11) the introduction of the Quality and Outcomes Framework. Although the scheme started in April 2004, practices had been aware of its broad outline since early 2003, and we therefore allowed for a possible "anticipation effect" in the preparatory year, 2003/04. There were large absolute differences in the rates of admission for incentivised ACSCs, non-incentivised ACSCs, and non-ACSCs. We analysed the inverse hyperbolic sine transformation of the admission rates, which can be interpreted in the same way as the logarithmic transformation but can be used when the dependent variable can take zero values. Therefore we estimated the effect of the Quality and Outcomes Framework on the proportionate, rather than on the absolute, differences between admission rates for incentivised ACSCs and the other two groups of conditions.

Before the introduction of the Quality and Outcomes Framework in 2004, admissions for incentivised ACSCs were increasing on average around 3\% per annum-less rapidly than for non-incentivised ACSCs and around $0.5 \%$ less rapidly than for non-ACSCs. In the absence of the Quality and Outcomes

Framework, if the difference in trends continued, incentivised ACSCs would have increased less than both non-incentivised ACSCs and non-ACSCs after 2004. We therefore allowed for differences in the underlying trends in admission rates between incentivised ACSCs and the control groups of admissions by fitting a linear annual trend to each series in the period before the introduction of the Quality and Outcomes Framework. We allowed family practices to have different initial admission rates when fitting these linear trends. We then calculated the trend adjusted admission rate for each of the three types of admission as the difference between the actual and predicted admission rate given the pre-Quality and Outcomes Framework trend for that series. We estimated the impact of the Quality and

Outcomes Framework scheme using the difference between the trend adjusted admission rate for incentivised ACSCs and the trend adjusted admission rate for non-incentivised ACSCs or non-ACSCs. Adjusting each series for the underlying trends leads to a more conservative estimate of the impact of the scheme.

Using the low income scheme index recorded in the first year of the Quality and Outcomes Framework scheme, we examined whether the trend adjusted differences in emergency hospital admissions for incentivised ACSCs relative to non-incentivised ACSCs and non-ACSCs were affected by the level of income deprivation in the practice population. The low income scheme index records the proportions of prescriptions in each practice that are dispensed without charge because the patient has a low income. We estimated the effect of the Quality and Outcomes Framework separately for practices with above and below median low income scheme index scores.

We conducted a supplementary analysis of admissions for incentivised ACSCs that does not involve comparison with a control group. We tested for differences in trends in admissions for incentivised ACSCs for the periods 1998/99 to 2002/03 (pre-Quality and Outcomes Framework) and 2004/05 to 2010/11 (after the introduction of the Quality and Outcomes Framework) using an interrupted time series analysis. ${ }^{30}$ The supplementary file gives full details of the methods.

We illustrated the financial implications of changes in admissions for incentivised ACSCs due to the Quality and Outcomes Framework for one financial year by applying the estimated change in the admission rate between 2003/04 and $2010 / 11$ to the admission rate in the final year (2003/04) of the pre-Quality and Outcomes Framework period for a population of 53 million for England in mid-2011, ${ }^{31}$ and assuming an average cost per ACSC admission of $£ 1739 .{ }^{32}$ All analyses were performed using STATA v13.

\section{Results \\ Changes in rates of emergency admissions}

Over the study period the emergency admission rate for all conditions increased by $34 \%$, from 637.0 per 10000 person years in $1998 / 99$ to 852.6 per 10000 person years in 2010/11. Admission rates increased by $39 \%$ (from 33.7 per 10000 person years in $1998 / 99$ to 46.8 per 10000 person years in 2010/11) for non-incentivised ACSCs and by $41 \%$ (from 516.4 per 10 000 person years in 1998/99 to 727.6 per 10000 person years in 2010/11) for non-ACSCs. The emergency admission rate decreased by $10 \%$ (from 87.0 per 10000 person years to 78.2 per 10000 person years) for incentivised ACSCs over the same period. Over the study period, ACSCs accounted for $16 \%$ of all emergency admissions, of which $67 \%$ were for incentivised conditions and $33 \%$ were for non-incentivised conditions.

The emergency admission rate before the introduction of the Quality and Outcomes Framework was increasing at a rate of $5.0 \%$ per annum (95\% confidence interval $4.5 \%$ to $5.5 \%$ ) for non-incentivised ACSCs and $4.2 \%$ per annum (3.9\% to $4.6 \%$ ) for non-ACSCs (fig $1 \Downarrow$ and supplementary table A1). In contrast, the rate of emergency admissions for incentivised ACSCs was increasing at a rate of only $1.7 \%$ (1.2\% to $2.2 \%)$ per annum over the same period.

\section{Estimates of effect of introduction of pay for performance scheme}

The table $\Downarrow$ summarises the percentage differences between the trend adjusted admission rates for the incentivised and non-incentivised ACSCs and non-ACSCs and these are plotted in figure $2 \Downarrow$. In the preparatory year for the Quality and Outcomes Framework (2003/04), the difference between the trend adjusted admission rates for incentivised and non-incentivised ACSCs was not significantly different from zero. However, in the first full year of the Quality and Outcomes Framework scheme (2004/05), when practice performance was measured and incentivised, the trend adjusted admission rate for incentivised ACSCs was $2.7 \%$ (95\% confidence interval $1.6 \%$ to $3.8 \%$ ) less than the trend adjusted admission rate for non-incentivised ACSCs. By the seventh year of the Quality and Outcomes Framework scheme (2010/11), the trend adjusted rate for incentivised ACSCs was 8.0\% (6.9\% to 9.1\%) less than for non-incentivised ACSCs. The trend adjusted admission rate for the incentivised ACSCs also decreased relative to the trend adjusted rate for non-ACSCs, from 2.8\% (2.0\% to 3.6\%) less in the first year of the Quality and Outcomes Framework scheme (2004/05) to $10.9 \%$ ( $10.1 \%$ to $11.7 \%)$ less by the seventh year (2010/11).

\section{Income deprivation}

The effect of the Quality and Outcomes Framework scheme on trend adjusted emergency admissions was similar for practices with above or below median values for income deprivation (see supplementary tables $\mathrm{A} 2$ and $\mathrm{A} 3$ and figures $\mathrm{A} 1$ and $\mathrm{A} 2$ ). There was a larger reduction in trend adjusted admission rates for incentivised ACSCs than for non-incentivised ACSCs for practices with above average values for income deprivation, although the difference was only statistically significant in 2010/11. 


\section{Supplementary analysis}

Interrupted time series analysis of admission rates for incentivised ACSCs, without comparison with other types of emergency admissions, showed that they were increasing in the pre-Quality and Outcomes Framework period (see supplementary table A4). In the preparatory year (2003/04) the increase was $2.5 \%$. After the introduction of the Quality and Outcomes Framework in 2004/05, the admission rate for incentivised ACSCs decreased at a rate of $3.6 \%$ per year (see supplementary figure A3). Comparing the actual trend in admissions from 2004/05 to 2010/11 with the projected trend from the pre-Quality and Outcomes Framework period, the estimated impact of the scheme was to reduce emergency admissions for incentivised ACSCs in 2010/11 by $16.6 \%$ (95\% confidence interval $13.5 \%$ to $19.5 \%$ ).

\section{Estimated impact on overall utilisation and cost}

The estimated reduction in admissions for incentivised ACSCs associated with the introduction of the Quality and Outcomes Framework scheme was $8 \%$ in the financial year 2010/11, equivalent to a reduction of approximately 53000 emergency admissions across England. This is a potential cost saving of around $£ 92.5 \mathrm{~m}$ ( $\$ 149 \mathrm{~m}$; $€ 117 \mathrm{~m})$ per year. The estimated effect from the interrupted time series analysis is a reduction of approximately 75500 emergency admissions in the financial year $2010 / 11$, which is a saving of $£ 131.5 \mathrm{~m}$.

\section{Discussion}

Our results show a moderate and sustained reduction in emergency hospital admissions for ambulatory care sensitive conditions (ACSCs) that were incentivised under the UK's Quality and Outcomes Framework pay for performance scheme. This reduction was $2.7 \%$ in the first year of the scheme, increasing to $8.0 \%$ in the seventh year. Our supplementary analysis, which considered only the underlying trend in admissions for the incentivised ACSCs, showed a larger reduction, which also coincided with the introduction of the Quality and Outcomes Framework scheme.

\section{Strengths and weaknesses of this study}

Our study is observational and subject to several limitations. Firstly, although hospital episode statistics records all emergency admissions to the NHS, any changes in the consistency and accuracy of data recording during the study period would affect our findings, particularly if incentivised conditions were less often recorded as the primary reason for admission from 2004. Secondly, we focused on non-incentivised conditions defined as ambulatory care sensitive by the NHS, and this limits generalisability of the study in countries that use different definitions. However, there is broad agreement across systems on the core group of ambulatory sensitive conditions, and our supplementary interrupted times series analysis-focusing solely on trends for incentivised conditions-produced a similar result to that of the main analysis. Thirdly, admission trends for incentivised and non-incentivised conditions were divergent before the introduction of the pay for performance scheme. This violates the assumption of parallel trends required for a simple difference in difference analysis, and we therefore adjusted admission rates for underlying trends in the pre-Quality and Outcomes Framework period. As a result, our estimate of the effect of the Quality and Outcomes Framework scheme in the main analysis is likely to be conservative. Finally, as the Quality and Outcomes Framework is a national intervention implemented by almost all family practices, there are no control practices and we therefore could not test our assumption that pre-intervention admission trends would have continued in the absence of incentives.

\section{Effect of primary care on hospital admissions}

Estimates from previous studies of the proportion of hospital admissions that can potentially be avoided with appropriate ambulatory care range from 5\% to $79 \% .{ }^{33}$ In our study, $16 \%$ of emergency admissions were designated as sensitive to ambulatory care. The empirical evidence on the effect of improving quality of primary care on emergency admissions is mixed, and several studies have found little or no association between quality of care and admissions. ${ }^{34-37}$ Previous studies on the Quality and Outcomes Framework scheme have found associations between improved primary care management and reduced emergency admissions for chronic obstructive pulmonary disease, ${ }^{38}$ coronary heart disease,${ }^{39}$ and stroke ${ }^{40}$ but not for hypertension. ${ }^{41}$ For diabetes, improved glycaemic control in people with diabetes is associated with fewer emergency admissions for hyperglycaemia without any increase in admissions for hypoglycaemia. ${ }^{29}$

\section{Effect of incentives in primary care on hospital admissions}

We found that the trend adjusted admission rate for ACSCs included in the Quality and Outcomes Framework fell by $2.7 \%$ in the first year of the scheme relative to the rate for ACSCs that were not included. This increased to $8 \%$ by the seventh year, equivalent to an annual reduction in emergency admissions of 53000 nationally. This result cannot easily be attributed to measured improvements in process indicators that occurred after the introduction of the Quality and Outcomes Framework scheme, as previous studies suggest that these were generally modest ${ }^{3}$ and would therefore be unlikely to explain the rapid change in admissions observed in our analysis. Furthermore, the improvements that did occur were greater for indicators relating to measurement (for example, blood pressure recording) than to intermediate outcomes ${ }^{42}$ or treatments, ${ }^{7}$ which would be more likely to have a rapid impact on admissions. Although the Quality and Outcomes Framework has the potential to have a major impact on health outcomes, ${ }^{43}$ in our view the improvements that have occurred under the scheme for indicators likely to have an immediate impact on admissions have not been sufficient for us to attribute the observed decrease in admissions solely to the Quality and Outcomes Framework scheme. We reached a similar conclusion in our analysis of a hospital based pay for performance scheme (the UK's advancing quality programme), where we suggested that observed improvements in patient outcomes were partly attributable to unmeasured changes associated with the introduction of pay for performance, rather than to changes in incentivised process measures. $^{17}$

\section{Other system changes affecting admissions}

Several other changes within the NHS during the study period impacted on hospital admissions. For example, in 2004 a target for patients to wait for no more than four hours in emergency departments; in 2004 a change to the national contract for family practices that allowed them to opt-out of providing out of hours care (most practices did so); and in 2005/06 a change in the method of payment for hospitals, from a block contract system that paid hospitals a fixed amount regardless of volume to a system that paid hospitals for each patient seen or treated. Each 
of these changes contributed to the overall increase in emergency admissions observed over the study period and will have biased our results if they had differential impacts on admissions for incentivised and non-incentivised conditions. For example, the introduction of the four hour wait target resulted in thousands of additional patients being admitted to hospital to avoid breaching the target, who would otherwise have been dealt with within emergency departments and discharged. ${ }^{44}$ Our results will have been affected if the chances of such a "breach" admission are greater for patients presenting with, for example, dehydration than for patients presenting with cardiac chest pain.

Admissions for heart disease and stroke accounted for $50 \%$ of admissions for incentivised ACSCs over the study period, and reductions in risk factors such as smoking generated secular trends, which we controlled for in our analysis. Several other quality improvement initiatives specifically tackling these conditions were implemented during our study period. National standards for the management of coronary heart disease in England were introduced in $2000^{45}$ and included a recommendation for the establishment of a national network of rapid access chest pain clinics that were rolled out between 2002 and 2006. Guidance from the National Institute for Health and Care Excellence was also issued on the management of myocardial infarction in 2001 (www.nice.org.uk), and British Hypertension Society guidelines on the management of hypertension were issued in 1999 and 2004 (www.bhsoc.org) followed by NICE guidance on hypertension in 2006. NICE guidance was also issued on the management of heart failure in 2003. Some of these might have impacted on the early changes we observed in the study, but none could readily be linked to the apparent sharp drop in admissions observed from 2004/05 onwards.

One potential explanation is that previous interventions that might have influenced patterns of admission were reinforced with the introduction of the Quality and Outcomes Framework scheme. If so, some of the changes in admission patterns that we observed could have been directly due to the Quality and Outcomes Framework (for example, better blood pressure control in stroke) and some may have been related to increased sensitivity to previous clinical guidelines (for example, the importance of early referral for transient ischaemic attack). This would be consistent with other research on quality improvement, which suggests that there is no "magic bullet" to improving quality: rather, interventions need to be multifaceted and sustained over time to produce major change. ${ }^{46}$

\section{Conclusions and policy implications}

Improvements in preventive medicine and disease management in primary care offer the hope of better patient outcomes and reduced demand for secondary care, bringing benefits for both health and healthcare expenditure. The UK's Quality and Outcomes Framework pay for performance scheme was introduced with the expectation that financial and reputational incentives (public reporting of performance allowing comparison between providers) would promote high quality primary care, improve patient outcomes, and reduce emergency admissions to secondary care. We found relative reductions in rates of emergency admissions for conditions incentivised under the Quality and Outcomes Framework scheme, particularly for coronary heart disease and stroke. The potential costs savings from these avoided admissions $(£ 92.5 \mathrm{~m}(\$ 149 \mathrm{~m} ; € 117 \mathrm{~m})$ by 2010/11) may seem small in comparison to the overall cost of the scheme (£1bn per annum), but it is important to note that benefits other than reduced admissions were expected from the scheme, including improved use of clinical computing systems and substantial uplifts in family practitioners' income to encourage recruitment and retention in the specialty.

Past research has focused on the direct consequences of financial incentives to improve quality and on the unexpected negative consequences of such incentives. Our results suggest that further research should also be carried out on the potential long term benefits and harms of pay for performance schemes on health outcomes.

Contributors: All authors were involved in the study design, analysis and interpretation of the data, and drafting and final approval of the manuscript. They accept full responsibility for the research, had access to the data, and controlled the decision to publish. MR is the guarantor of the study.

Funding: This study received no direct funding, but it was initiated at a time when the National Primary Care Research and Development Centre at the University of Manchester was receiving core funding from the UK Department of Health. The views expressed are those of the authors and not necessarily those of the Department of Health. The researchers are totally independent of the funders.

Competing interests: All authors have completed the ICMJE uniform disclosure form at www.icmje.org/coi_disclosure.pdf (available on request from the corresponding author) and declare: no financial relationships with any organisations that might have an interest in the submitted work in the previous three years; MR served as an academic advisor to the government and British Medical Association, negotiating teams during the development of the UK pay for performance scheme during 2001 and 2002.

Ethical approval: Not required.

Data sharing: The statistical code is available on request from $\mathrm{MH}$ (mark. harrison@ubc.ca). The dataset was derived from hospital episode statistics, access to which requires permission from the Health and Social Care Information Centre.

Transparency: The guarantor (MR) affirms that this manuscript is an honest, accurate, and transparent account of the study being reported; that no important aspects of the study have been omitted; and that any discrepancies from the study as planned (and, if relevant, registered) have been explained.

Rosenthal MB, Frank RG, Li Z, Epstein AM. Early experience with pay-for-performance: from concept to practice. JAMA 2005;294:1788-93.

2 Damberg CL, Raube K, Teleki SS, Dela CE. Taking stock of pay-for-performance: a candid assessment from the front lines. Health Aff (Millwood) 2009;28:517-25.

3 Campbell SM, Reeves D, Kontopantelis E, Sibbald B, Roland M. Effects of pay for performance on the quality of primary care in England. N Engl J Med 2009;361:368-78.

4 Sutton M, Elder R, Guthrie B, Watt G. Record rewards: the effects of targeted quality incentives on the recording of risk factors by primary care providers. Health Econ 2010;19:1-13.

5 Emmert M, Eijkenaar F, Kemter H, Esslinger AS, Schoffski O. Economic evaluation of pay-for-performance in health care: a systematic review. Eur $\mathrm{J}$ Health Econ 2012;13:755-67.

6 Kruse GB, Polsky D, Stuart EA, Werner RM. The impact of hospital pay-for-performance on hospital and Medicare costs. Health Serv Res 2012;47:2118-36.

7 Mitchell C, Dwyer R, Hagan T, Mathers N. Impact of the QOF and the NICE guideline in the diagnosis and management of depression: a qualitative study. Br J Gen Pract 2011;61:e279-89.

8 Doran T, Kontopantelis E, Valderas JM, Campbell S, Roland M, Salisbury C, et al. Effect of financial incentives on incentivised and non-incentivised clinical activities: Iongitudinal analysis of data from the UK Quality and Outcomes Framework. BMJ 2011;342:d3590.

9 Christianson J, Leatherman S, Sutherland K. Financial incentives, healthcare providers and quality improvements: a review of the evidence. Health Foundation, 2009.

10 Gillam SJ, Siriwardena AN, Steel N. Pay-for-performance in the United Kingdom: impact of the quality and outcomes framework: a systematic review. Ann Fam Med 2012:10:461-8.

11 Flodgren G, Eccles MP, Shepperd S, Scott A, Parmelli E, Beyer FR. An overview of reviews evaluating the effectiveness of financial incentives in changing healthcare professional behaviours and patient outcomes. Cochrane Database Syst Rev 2011;7:CD009255

12 Chen JY, Tian H, Taira JD, Hodges KA, Brand JC, Chung RS, et al. The effect of a PPO pay-for-performance program on patients with diabetes. Am J Manag Care 2010;16:e11-9.

13 Fiorentini G, lezzi E, Lippi BM, Ugolini C. Incentives in primary care and their impact on potentially avoidable hospital admissions. Eur J Health Econ 2011;12:297-309.

14 Martin S, Smith P, Gravelle H, Rice N. Do quality improvements in primary care reduce secondary care costs? Health Foundation, 2011.

15 Chen JY, Tian H, Juarez DT, Yermilov I, Braithwaite RS, Hodges KA, et al. Does pay for performance improve cardiovascular care in a "real-world" setting? Am J Med Qual 2011;26:340-8. 


\section{What is already known on this topic}

Pay for performance schemes in healthcare, which link provider payments to quality of care, are associated with improvements in recorded processes of care

Evidence on the impact of pay for performance schemes on health outcomes is mixed, and several studies have found little or no impact

\section{What this study adds}

The UK's Quality and Outcomes Framework pay for performance scheme for primary care was associated with a decrease in emergency admissions for incentivised conditions compared with conditions that were not incentivised under the scheme

The change seems larger than could be explained by changes in the incentivised process measures, suggesting that the scheme may have had unmeasured impacts on quality of care beyond the directly incentivised activities

Further research into the potential long term benefits and harms of pay for performance schemes on health outcomes is needed

16 Chang RE, Lin SP, Aron DC. A pay-for-performance program in Taiwan improved care for some diabetes patients, but doctors may have excluded sicker ones. Health Aff (Millwood) 2012;31:93-102.

17 Sutton M, Nikolova S, Boaden R, Lester H, McDonald R, Roland M. Reduced mortality with hospital pay for performance in England. N Engl J Med 20128;367:1821-8.

18 Houle SK, McAlister FA, Jackevicius CA, Chuck AW, Tsuyuki RT. Does performance-based remuneration for individual health care practitioners affect patient care?: a systematic review. Ann Intern Med 201218;157:889-99.

19 Werner RM, Dudley RA. Making the 'pay' matter in pay-for-performance: implications for payment strategies. Health Aff (Millwood) 2009 September;28(5):1498-508.

20 Conrad DA, Perry L. Quality-based financial incentives in health care: can we improve quality by paying for it? Annu Rev Public Health 2009;30:357-71.

21 Van HP, De SD, Annemans L, Remmen R, Rosenthal MB, Sermeus W. Systematic review: effects, design choices, and context of pay-for-performance in health care. BMC Health Serv Res 2010;10:247.

22 Roland M. Pay-for-performance: not a magic bullet. Ann Intern Med 2012;157:912-3.

23 Chernew ME, Mechanic RE, Landon BE, Safran DG. Private-payer innovation in Massachusetts: the 'alternative quality contract'. Health Aff (Millwood) 2011;30:51-61.

24 Song Z, Safran DG, Landon BE, He Y, Ellis RP, Mechanic RE, et al. Health care spending and quality in year 1 of the alternative quality contract. N Engl J Med 2011;365:909-18.

25 Blunt I, Bardsley M, Dixon J. Trends in emergency admissions in England 2004-2009. Nuffield Trust; 2010.

26 Roland M. Linking physicians' pay to the quality of care-a major experiment in the United Kingdom. N Engl J Med 2004:351:1448-54.

27 Roland M, Campbell S. Successes and failures of the United Kingdom's pay for performance program. N Engl J Med 2014;370:1944-9.

28 Purdy S, Griffin T, Salisbury C, Sharp D. Ambulatory care sensitive conditions: terminology and disease coding need to be more specific to aid policy makers and clinicians. Public Health 2009;123:169-73.

29 Dusheiko M, Doran T, Gravelle H, Fullwood C, Roland M. Does higher quality of diabetes management in family practice reduce unplanned hospital admissions? Health Serv Res 2011;46(1 Pt 1):27-46

30 Wagner AK, Soumerai SB, Zhang F, Ross-Degnan D. Segmented regression analysis of interrupted time series studies in medication use research. $J$ Clin Pharm Ther 2002;27:299-309

31 Office for National Statistics. Population estimates for England and Wales, mid-2011 (2011 Census-based). ONS, 2012

32 Tian $\mathrm{Y}$, Dixon A, Gao H. Emergency hospital admissions for ambulatory care-sensitive conditions: identifying the potential for reductions. King's Fund, 2012.

33 Van Walraven C, Bennett C, Jennings A, Austin PC, Forster AJ. Proportion of hospita readmissions deemed avoidable: a systematic review. CMAJ 2011;183:E391-E402.

34 Durojaiye LI, Hutchison T, Madeley RJ. Improved primary care does not prevent the admission of children to hospital. Public Health 1989;103:181-8.

35 Weinberger M, Oddone EZ, Henderson WG. Does increased access to primary care reduce hospital readmissions? Veterans Affairs Cooperative Study Group on Primary Care and Hospital Readmission. N Engl J Med 1996;334:1441-7.
36 Bottle A, Gnani S, Saxena S, Aylin P, Mainous AG, Majeed A. Association between quality of primary care and hospitalization for coronary heart disease in England: National cross-sectional study. J Gen Intern Med 2008;23:135-41.

37 Purdy S, Griffin T, Salisbury C, Sharp D. Emergency respiratory admissions: influence of practice, population and hospital factors. J Health Serv Res Policy 2011;16:133-40.

38 Calderon-Larranaga A, Carney L, Soljak M, Bottle A, Partridge M, Bell D, et al. Association of population and primary healthcare factors with hospital admission rates for chronic obstructive pulmonary disease in England: national cross-sectional study. Thorax 2011;66:191-6.

39 Kiran T, Hutchings A, Dhalla IA, Furlong C, Jacobson B. The association between quality of primary care, deprivation and cardiovascular outcomes: a cross-sectional study using data from the UK Quality and Outcomes Framework. J Epidemiol Community Health 2010;64:927-34.

40 Soljak M, Calderon-Larrañaga A, Sharma P, Cecil E, Bell D, Abi-Aad G, et al. Does higher quality primary health care reduce stroke admissions? A national cross-sectional study. Br J Gen Pract 2011;61:e801-7.

41 Serumaga B, Ross-Degnan D, Avery AJ, Elliott RA, Majumdar SR, Zhang F, et al. Effect of pay for performance on the management and outcomes of hypertension in the United Kingdom: interrupted time series study. BMJ 2011;342:d108.

42 Simpson CR, Hannaford PC, Ritchie LD, Sheikh A, Williams D. Impact of the pay-for-performance contract and the management of hypertension in Scottish primary care: a 6-year population-based repeated cross-sectional study. Br J Gen Pract 2011;61:e443-51.

43 Fleetcroft R, Parekh-Bhurke S, Howe A, Cookson R, Swift L, Steel N. The UK pay-for-performance programme in primary care: estimation of population mortality reduction. Br J Gen Pract 2010;60:e345-52.

44 National Audit Office. Emergency admissions to hospital: managing the demand. Stationery Office, 2013.

45 Department of Health. Department of Health National Framework for Coronary Heart Disease. DoH, 2000.

46 Oxman AD, Thomson MA, Davis DA, Haynes RB. No magic bullets: a systematic review of 102 trials of interventions to improve professional practice. CMAJ 1995;153:1423-31.

\section{Accepted: 14 October 2014}

\section{Cite this as: BMJ 2014;349:96423}

This is an Open Access article distributed in accordance with the Creative Commons Attribution Non Commercial (CC BY-NC 4.0) license, which permits others to distribute, remix, adapt, build upon this work non-commercially, and license their derivative works on different terms, provided the original work is properly cited and the use is non-commercial. See: http://creativecommons.org/licenses/by-nc/4.0/. 


\section{Table}

Table 1| Estimates of effect of Quality and Outcomes Framework (QOF): percentage differences between trend adjusted emergency admission rates for incentivised and non-incentivised ambulatory care sensitive conditions (ACSCs)

\begin{tabular}{|c|c|c|c|c|}
\hline \multirow[b]{2}{*}{ Period and year } & \multicolumn{2}{|c|}{ Control group: non-incentivised ACSCs } & \multicolumn{2}{|c|}{ Control group: non-ACSCs } \\
\hline & $\%$ difference ${ }^{*}(95 \% \mathrm{Cl})$ & $P$ value & $\%$ difference $^{*}(95 \% \mathrm{Cl})$ & $P$ value \\
\hline \multicolumn{5}{|l|}{ Pre-QOF: } \\
\hline 1998/99 & $-1.25(-2.36$ to -0.14$)$ & 0.028 & $0.38(-0.42$ to 1.18$)$ & 0.351 \\
\hline 1999/2000 & $2.08(0.96$ to 3.19$)$ & 0.000 & $1.25(0.45$ to 2.05$)$ & 0.002 \\
\hline $2000 / 01$ & $-0.69(-1.80$ to 0.43$)$ & 0.227 & $-2.71(-3.50$ to -1.91$)$ & 0.000 \\
\hline $2001 / 02$ & $0.14(-0.98$ to 1.25$)$ & 0.809 & $0.14(-0.65$ to 0.94$)$ & 0.725 \\
\hline $2002 / 03$ & $-0.28(-1.39$ to 0.83$)$ & 0.622 & $0.93(0.14$ to 1.73$)$ & 0.022 \\
\hline \multicolumn{5}{|l|}{ Preparatory: } \\
\hline $2003 / 04$ & $-0.01(-1.12$ to 1.10$)$ & 0.983 & $-0.24(-1.03$ to 0.56$)$ & 0.561 \\
\hline \multicolumn{5}{|l|}{ Post-QOF: } \\
\hline $2004 / 05$ & $-2.68(-3.79$ to -1.57$)$ & 0.000 & $-2.78(-3.58$ to -1.99$)$ & 0.000 \\
\hline $2005 / 06$ & $-6.98(-8.09$ to -5.87$)$ & 0.000 & $-8.21(-9.01$ to -7.41$)$ & 0.000 \\
\hline $2006 / 07$ & $-7.66(-8.78$ to -6.55$)$ & 0.000 & $-8.65(-9.45$ to -7.85$)$ & 0.000 \\
\hline $2007 / 08$ & $-10.51(-11.63$ to -9.40$)$ & 0.000 & $-11.33(-12.13$ to -10.53$)$ & 0.000 \\
\hline $2008 / 09$ & $-7.53(-8.64$ to -6.41$)$ & 0.000 & $-10.50(-11.30$ to -9.70$)$ & 0.000 \\
\hline $2009 / 10$ & $-11.00(-12.12$ to -9.89$)$ & 0.000 & $-13.99(-14.79$ to -13.19$)$ & 0.000 \\
\hline $2010 / 11$ & $-7.98(-9.09$ to -6.87$)$ & 0.000 & $-10.94(-11.74$ to -10.15$)$ & 0.000 \\
\hline
\end{tabular}

*Percentage difference between trend adjusted admission rate for incentivised ACSCs and trend adjusted admission rates for non-incentivised ACSCs and for non-ACSCs. Trend adjusted admission rate is logarithm of admission rate for year minus logarithm of admission rate predicted from fitting a linear trend for pre-QOF period $1998 / 99$ to $2002 / 03$. 


\section{Figures}

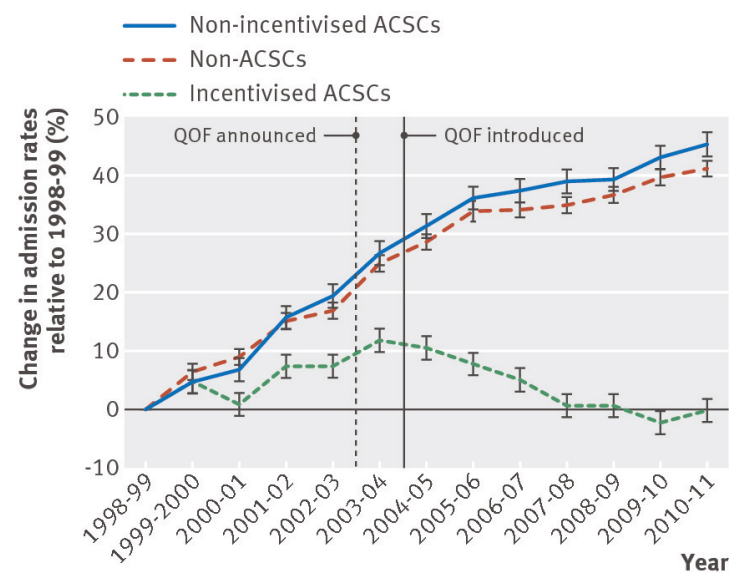

Fig 1 Percentage change in emergency admissions relative to 1998/99 with no adjustment for trend. ACSC=ambulatory care sensitive conditions; QOF=Quality and Outcomes Framework

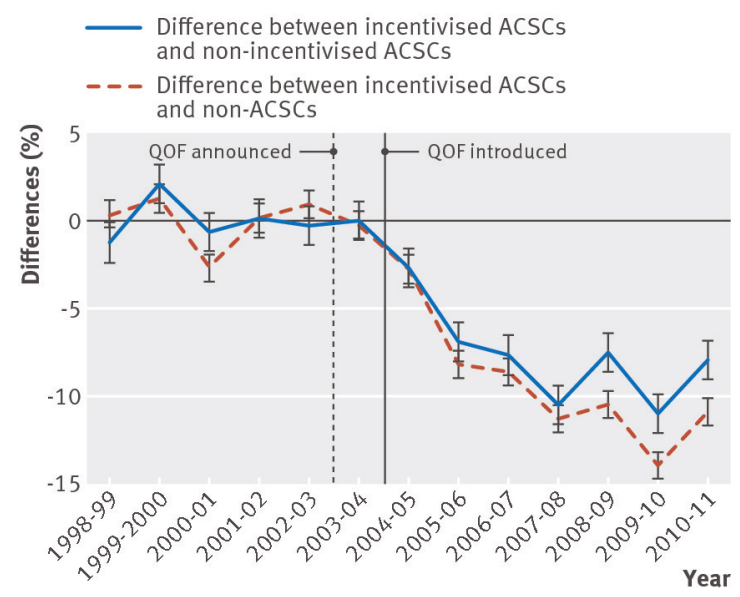

Fig 2 Percentage differences between trend adjusted emergency admission rates for incentivised ACSCs (ambulatory care sensitive conditions) and non-incentivised ACSCs and non-ACSCs. The trend adjusted admission rate is the logarithm of the actual admission rate for the year minus the logarithm of the admission rate predicted from fitting a linear trend for the pre-Quality and Outcomes Framework (QOF) period 1998/99-2002/03 